\title{
Acupuncture main channels or meridians: visible surfaces
}

\begin{abstract}
Summary
Courses of main channels later known in the West as meridians were painted as lines on the human body in Chinese acupuncture charts from head and chest regions to hands and feet. However none faithfully follow paths taken by any known anatomical structures. However when the body is entirely covered in oil or some other reflective substance and posed in a similar manner to Ming dynasty drawings in a darkened room lit by only one light source, one can perceive reflections of light proceeding in much the same directions as those shown in acupuncture illustrations. This suggests they were drawn from observations of the living body rather than as yet unknown anatomical structures dissected after death.
\end{abstract}

Volume 7 Issue 4 - 2017

\author{
Alexander Macdonald \\ Bristol BS8 IBA, UK
}

Correspondence: Alexander Macdonald, Bristol BS8 IBA, UK, Email alexmac@lineone.net

Received: December 23, 2016 | Published: June 14, 2017

\section{Introduction}

Categories of channels or vessels were described by Chinese medical practitioners as 'main', 'branch', 'connecting', 'muscle', 'divergent' and 'extra'. The best known today are the main channels. Soulié de Morant ${ }^{1}$ who had served in the French diplomatic corps in China renamed them as 'Méridians' - see Figure1. Their pathways have attracted attention as they were drawn with such care on the surfaces of acupuncture mannequins and charts. When unusual sensations, called de $q i$, are produced by manipulating a deeply inserted acupuncture needle, the Research Group of Acupuncture Anesthesia, Institute of Medicine and Pharmacology of Fujian Province ${ }^{2}$ noted how $20 \%$ of 64,228 patients in twenty-eight hospitals in China described them as passing up and down short lengths of the course of a main channel when a needle is inserted anywhere along its length; while $0.4 \%$ described these sensations travelling much further distances almost all if not the whole of its length (Figure 2). When an abnormally tender region is needled, Western observers such as Travell ${ }^{3}$ noted pain is often referred to some distant location from the hip region for example to the ankle. When compared to acupuncture charts, Western drawings of these two sites (the tender and referred) involve many of the same parts of the body linked by Chinese main channels - see Figure 3: this idea that Chinese and Western practitioners who had no knowledge of each other's observations were describing aspects of the same phenomenon was first put forward by $\mathrm{Mann}^{3} \&$ Melzack, ${ }^{5}$ later supported by Macdonald ${ }^{6} \&$ Dorsher. ${ }^{7}$ Yet another explanation has been advanced by authors such as Langevin, ${ }^{8}$ Bai $^{9}$ $\& \mathrm{Tao}^{10}$ who suggested networks of deep fascia might have been the source of the directions taken by main channels, that as can be seen from Figure 1 do not faithfully pursue any other known anatomical structures: for example no continuous vascular, lymphatic or neural structure travels from the head or chest to the foot or hand, as these main channels are shown to do.

Schorrenberger ${ }^{11}$ described reports of drawings made by the coroner of dissections of the human body carried out by Chinese doctors following the execution of bandits during the Northern Song Dynasty (AD 960-1127); as Wong ${ }^{12}$ describes, in ancient times doctors were not often encouraged to perform anatomical dissections, particularly as patients did not wish to leave their bodies for dissection. As Confucius said, "Our body with skin and hair comes from our parents. We must not mutilate it".

Could there have been another reason altogether why Chinese practitioners took the trouble to illustrate these particular surfaces of the human body?

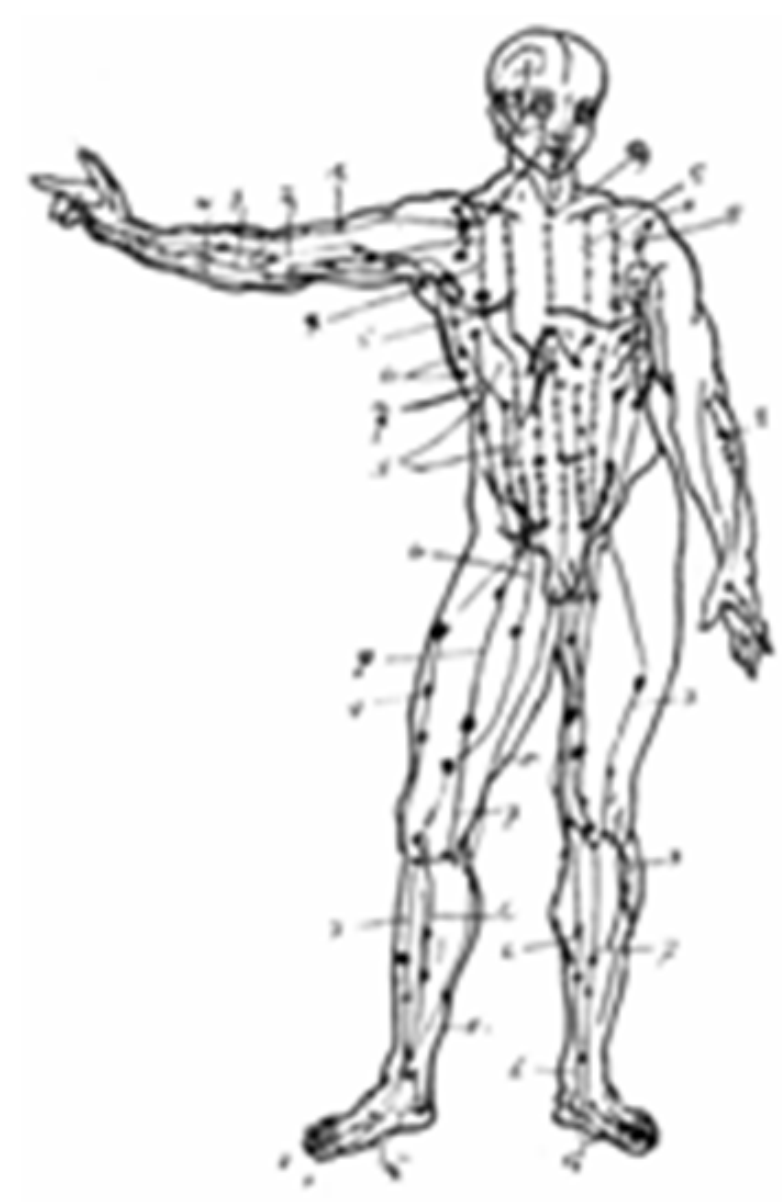

Figure I 'Les méridiens'

The theory I would like to present here is that the impetus to perform such an intricate study of surfaces of the human body may have been in keeping with advances in other disciplines such as architecture, armour, art, clothing and various postures of the body recommended in exercises. Optical studies produced 'magic mirrors' and a camera obscura. Mathematics and astronomy had become so advanced navigators' explorations and forecasts of eclipses became possible. Stone gnomons built tall enough to cast shadows of the sun not only to indicate the passing of time from day to day but also the seasons of the year. By the $5^{\text {th }}$ Century $\mathrm{AD}$, the number of days in a 
year had been calculated to be 365.243 . To integrate their thoughts with such activities, did medical practitioners recommend a similar number of acupuncture points to be scattered as stars on the body connected by constellations of main channels? The earth's ecliptic passage around the sun, the 'yellow path', was divided into twelve houses. Does this Figure explain why the same number of main channels on each side of the body? Or was this more to do with the number of great rivers in China?

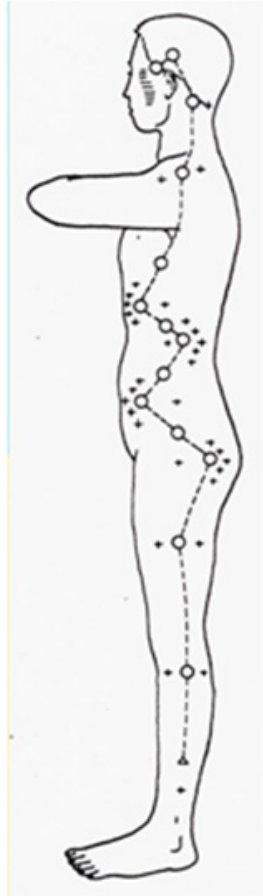

Figure 2 The path taken by unusual sensations called de qi when an acupuncture needle is manipulated in any site along its length - in this case the lateral shaoyang foot gallbladder main channel experienced by $0.4 \%$ of patients reported by the Research Group of Acupuncture Anesthesia, Institute of Medicine and Pharmacology of Fujian Province, 1986.

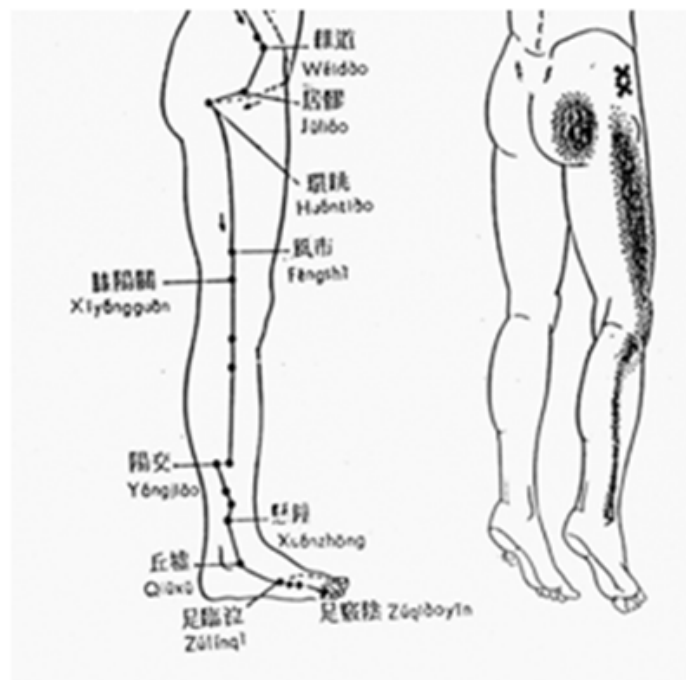

Figure 3 On the left side there is a Chinese illustration of the lateral shaoyang foot gallbladder main channel. On the right is a Western drawing by Travell et al 1992 showing a somewhat similar pattern of referred pain in many patients suffering an abnormally tender gluteus minimus muscle: here the referred pains shown in the shaded areas were caused by inserting needles into the tender gluteus minimus region at the sites indicated by two crosses.
Many practitioners of acupuncture dislike the geographical term 'meridian' being applied or mistranslated so recently by a European, Soulié de Morant. However this title might provide us with an idea. In geographical terms, lines of longitude flow over the surface of the earth from the North Pole to the South. Wherever one stands, there is a line of longitude beneath one's feet. The name 'meridian' (meridies, 'mid-day') occurs when the sun is directly overhead, as it is 'solar noon' not only here but everywhere else on that line however far north or south. Could each main channel link many parts of the body that under certain circumstances behave in a similar fashion to a line of longitude?

Unlike the geographical globe, the human body is composed of irregularly shaped structures whose cross-sections are not circular. Thus if the body happened to be presented in such a way that the surfaces of a particular main channel began to face the same way, their exact path within the body would not always be easy to predict. Indeed the irregularity of the structures of the human body might be the reason for the otherwise bizarre twists and turns the main channel pathways appear to take. Unlikely as this possibility may be, could this line of enquiry be one that the Chinese practitioners took such trouble to undertake?

\section{Surfaces of 'main channels' or 'meridians'}

The idea that the Chinese practitioners were interested in the direction surfaces of the body faced becomes more probable when one considers special names they gave. For example, while standing with one's back to the sun at noon, the anterior and medial surfaces tend to be kept in the shade; so these surfaces were titled yin. Conversely, the posterior and lateral surfaces of the body and limbs were exposed to the sun and were therefore called yang - see Figure 4. Furthermore as Figure 5 reveals, six surfaces of each limb were given special yin and yang titles: 1) the most shaded anteromedial surface was titled taiyin (great yin); 2) the less well shaded medial surface was called jeuyin (absolute yin); 3) being even less in the shade the posteromedial surface was said to be shaoyin (lesser yin); 4) the posterolateral surface received the most sun and was therefore called taiyang (great yang); 5) the lateral surface seeing somewhat less of the sun was given the title shaoyang (lesser yang); and finally 6) the anterolateral surface received even less exposure so was called yangming (sunlight yang).
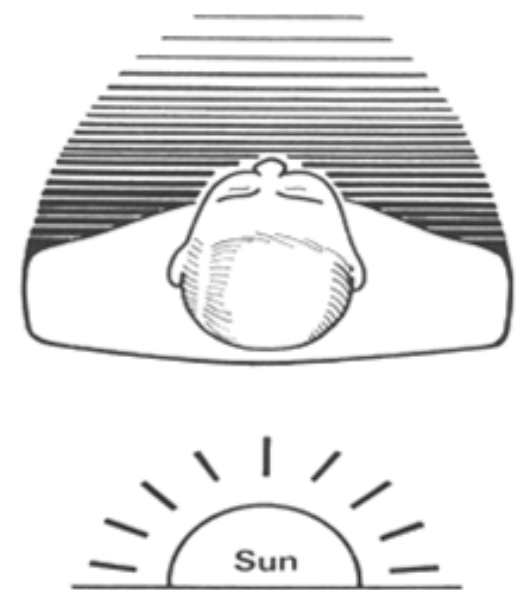

Figure $\mathbf{4}$ In relation to the sun at noon, the posterior and lateral surfaces are bathed in sunlight and therefore were considered to be yang, while the more shaded anterior and medial surfaces of the body tended to be described as yin. ${ }^{6}$ 


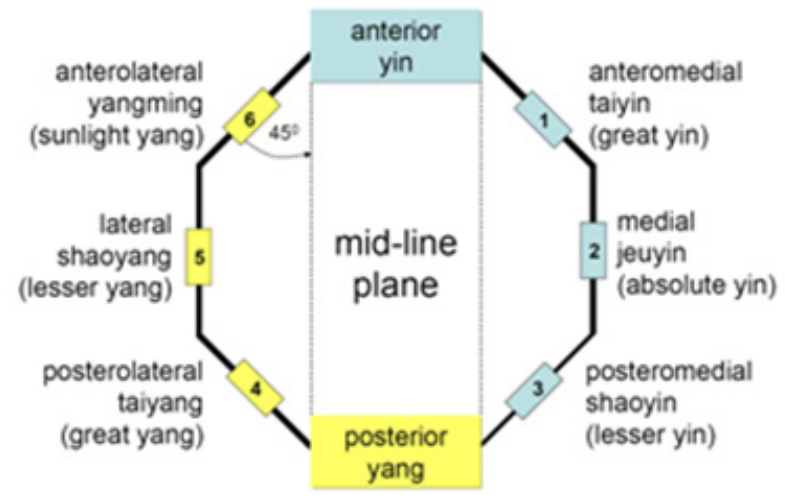

Figure 5 Here are Western and Chinese names for surfaces of a crosssection of a limb as seen from above. These surfaces are presented as sides of an octagon. Four oblique surfaces (I, 3, 4 and 6) are at forty-five degrees to the mid-line plane, whereas both the medial and lateral surfaces ( 2 and 5 ) run parallel to it.

In Figure 5,the tangents to the six numbered surfaces have been arranged on the sides of an imaginary octagon (a cross-sectional arrangement favoured in the building of some of the most beautiful pagodas and temples). Here the anterior and posterior surfaces of the octagon form a 'mid-line plane'. Running parallel to the mid-line plane, the second and fifth tangents occupied the lateral and medial positions. While all the other tangents were presented at forty-five degrees to the mid-line plane.

It may be helpful to see where tangents maintained at the same angles would have touched the skin of a cross-section of the human body. In Western anatomy, Figure $6^{13}$ revealed a section of the left leg taken a hand's breadth below the knee joint seen from above. Superimposed on this cross-section are sites where the six numbered tangents touch the skin when presented at the same angles to the midline plane as shown in Figure 5. It is worth noting the cross-section of the leg in Figure 6 is not circular; therefore sites where skin is touched by the six tangents are not arranged symmetrically. Furthermore this cross-section was made after death, so there is no circulation or normal muscle tone and the curvature of the skin will have changed accordingly.

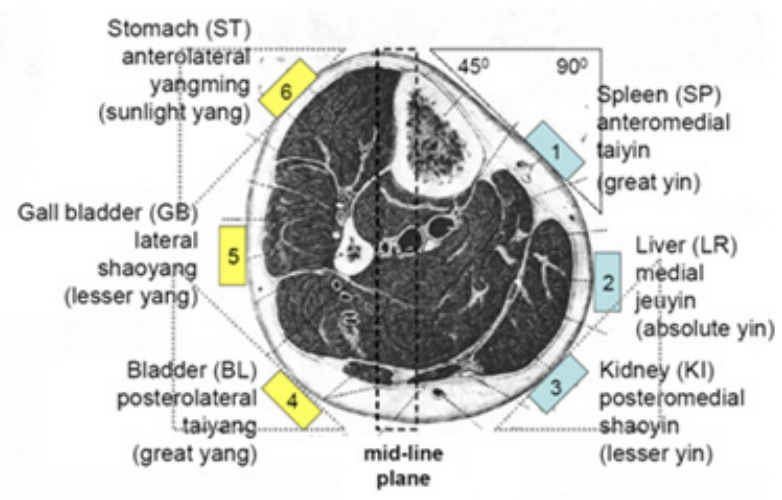

Figure 6 Section of the left leg $^{13}$ a hand's breadth below the knee joint is seen from above. It is suggested the most likely places for a surface of a 'main channel' or 'meridian' to occur are where tangents (at the same angles to the mid-line plane, presented in Figure 3) touch the skin.

\section{Hand and foot 'main channels' or 'meridians'}

We do not know why, but the Chinese not only allotted each of six surfaces of the upper and lower limbs to a main channel but also named it after one of twelve internal organs - see Table 1. In this table, organs in the second column were anatomically superior to the rest - so they were considered to be connected to the main channels of the hand: indeed they were referred to as 'hand main channels'. Meanwhile as the location of the organs in the third column of the table is more caudal, their main channels were designated 'foot main channels'.

Table I Six surfaces of upper and lower limbs together with the organs they were named after. The acronyms for each organ are taken from WHO I984

\begin{tabular}{|c|c|c|c|}
\hline \multicolumn{4}{|c|}{ Main Channels (Meridians) } \\
\hline S. No & Surfaces & Hand & Foot \\
\hline I & $\begin{array}{l}\text { Taiyin (Great Yin) } \\
\text { Anteromedial }\end{array}$ & Lung (LU) & Spleen (SP) \\
\hline 2 & $\begin{array}{l}\text { Jeuyin (Absolute Yin) } \\
\text { Medial }\end{array}$ & Pericardium (PE) & Liver (LR) \\
\hline 3 & $\begin{array}{l}\text { Shaoyin (Lesser Yin) } \\
\text { Posteromedial }\end{array}$ & Heart $(\mathrm{HT})$ & Kidney (KI) \\
\hline 4 & $\begin{array}{l}\text { Taiyang (Great Yang) } \\
\text { Posterolateral }\end{array}$ & Small Intestine (SI) & Bladder (BL) \\
\hline 5 & $\begin{array}{l}\text { Shaoyang (Lesser Yang) } \\
\text { Lateral }\end{array}$ & Triple Energizer (TE) & Gallbladder (GB) \\
\hline 6 & $\begin{array}{l}\text { Yangming (Sunlight Yang) } \\
\text { Anterolateral }\end{array}$ & Large Intestine (LI) & Stomach (ST) \\
\hline
\end{tabular}

Courses of hand and foot main channels are shown inFigure 7 A \& Bby Mann. ${ }^{14}$ One can see that each surface faces much the same way during its path in upper and lower limbs. Indeed to check the course of those surfaces that are forty-five degrees to the mid-line plane, one can employ an isosceles right triangle obtained by cutting a square block along its diagonal. Place this triangular structure on a limb in such a way that one side is kept perpendicular to the mid-line plane. As shown in Figure 6, wherever it first mak-..$^{15-18}$

\section{HAND 'MAIN CHANNELS' or 'MERIDIANS'}
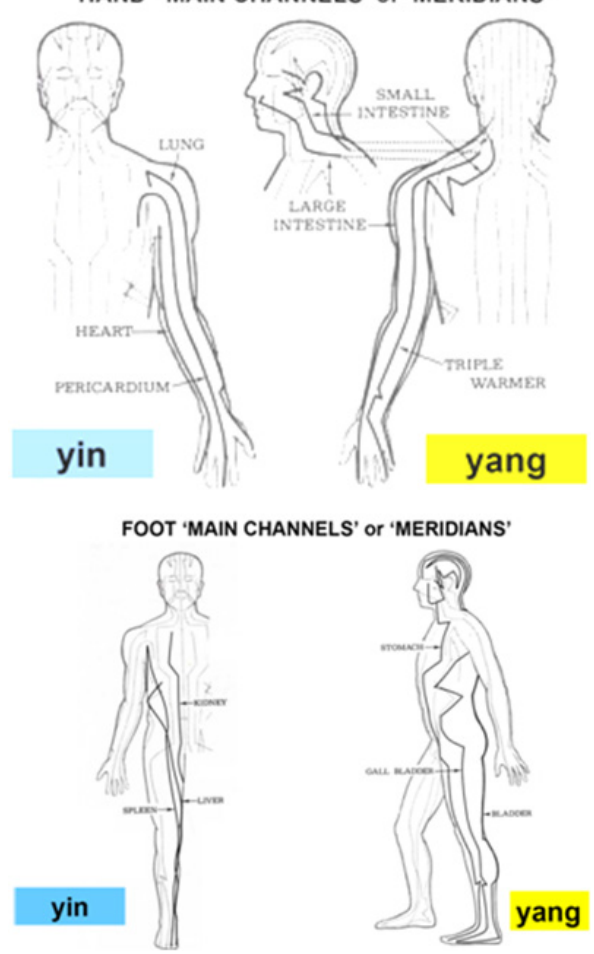

Figure 7 7(A) the hand and (B), the foot 'main channels' or 'meridians'14 


\section{Anatomical positions and other poses}

When attempting to discover the surfaces of main channels in Chinese medicine what was the 'anatomical position' of the subject? As this places the surfaces of the hand main channels in somewhat similar positions to those of the leg, it has been suggested the Chinese anatomical position may have required the forearm to be held halfway between pronation and supination, as shown in the life sized bronze replica of the human body originally cast in the $12^{\text {th }}$ Century $\mathrm{AD}$, see Figure8. However to show the paths taken by each main channel, a much bolder approach was taken. In Ming Dynasty drawings a different pose or anatomical position was recommended for each channel (Figures $9 \& 10$ ).

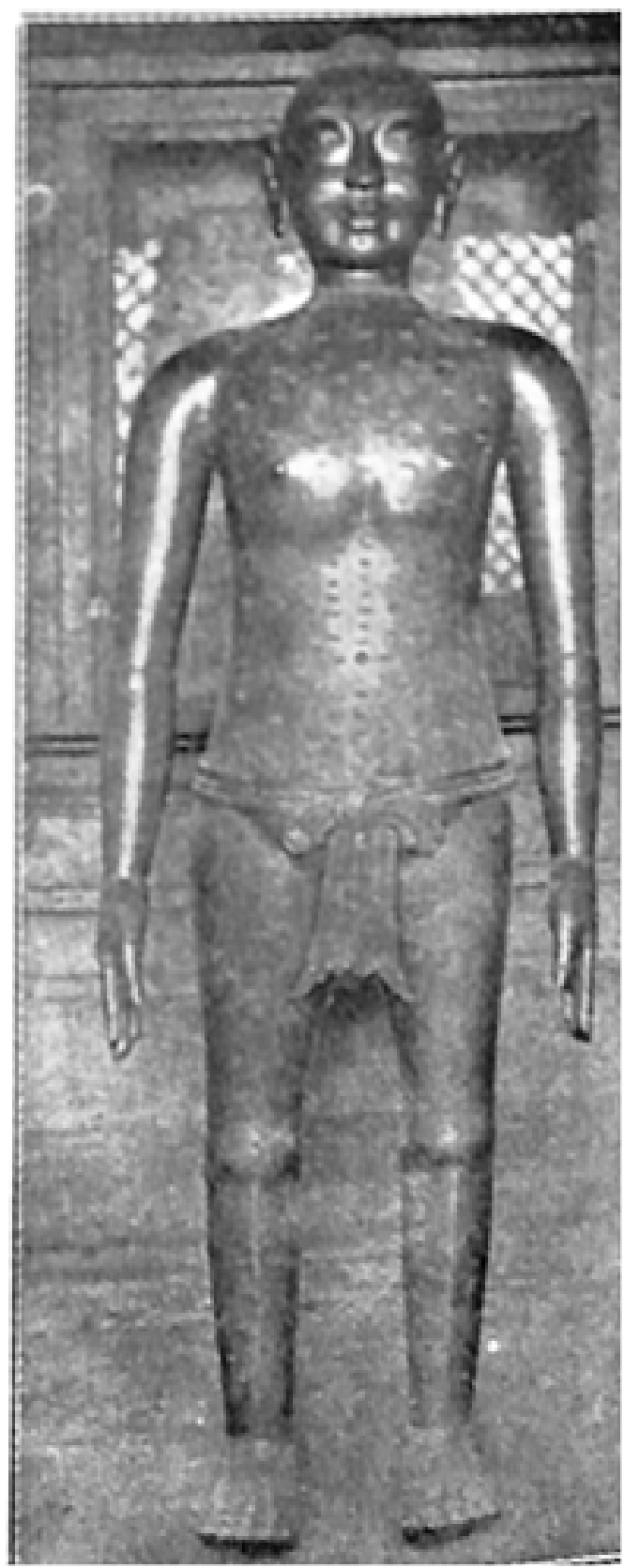

Figure 8 Photograph taken by DrWu Lien-Teh ${ }^{12}$ of a life sized bronze figure said to be a copy of one ordered by the Emperor to be cast by Wang Weiyi in 1027 AD to demonstrate the loci of acupuncture points. Note the highlights that have appeared on this photograph on the anterolateral, yangming (sunlight) surfaces on the face, trunk, upper and lower limbs.
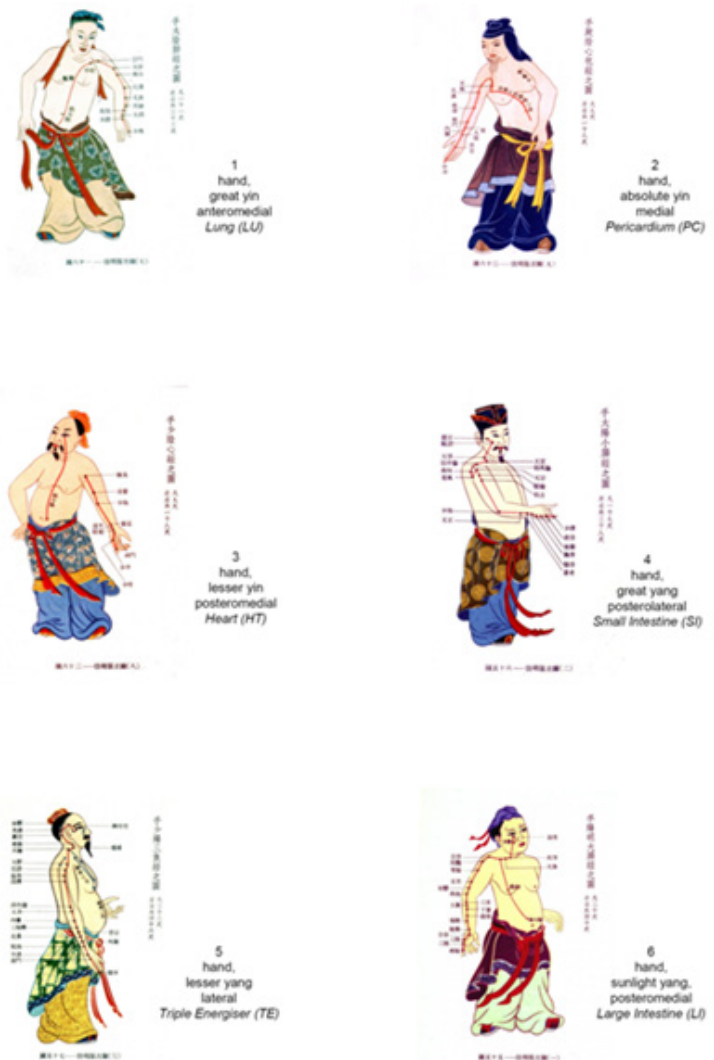

Figure 9 Ming Dynasty poses for each of the hand 'main channels' or 'meridians'.
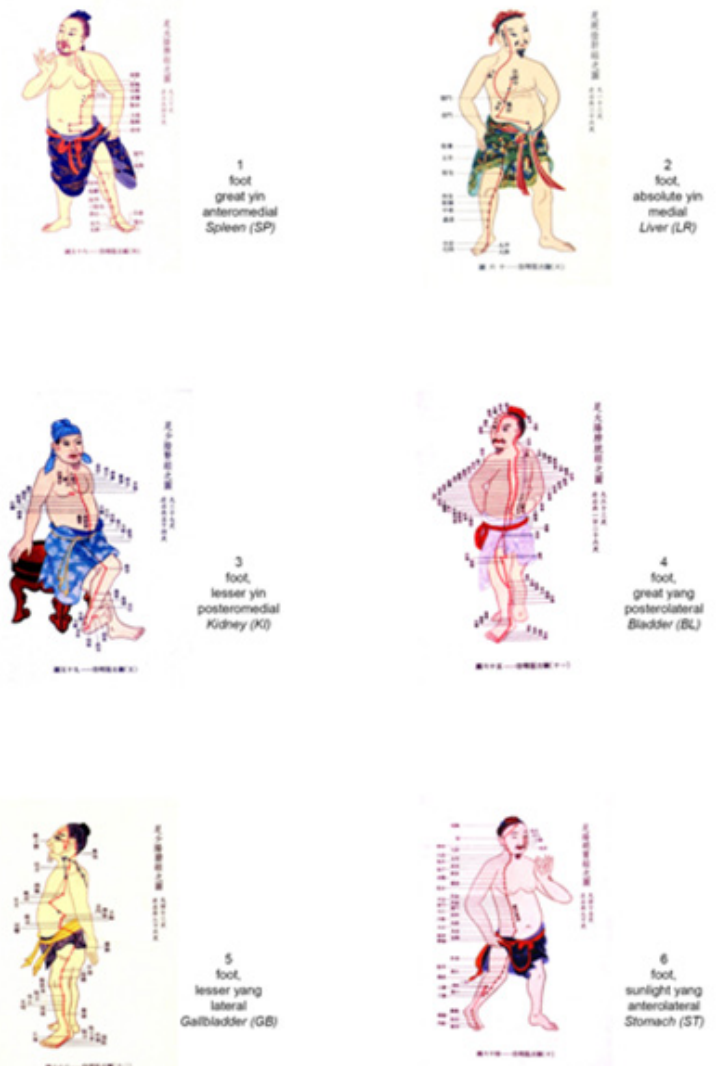

Figure 10 Ming dynasty poses for each of the foot 'main channels' or 'meridians'. 
If we take the Bladder foot main channel for example, at first sight, if the Figure was stood in the Western anatomical position, it does indeed pursue the posterolateral taiyang (great yang) surface at fortyfive degrees to the mid-line plane but only during its course in the lower limb. While standing in this way, the posterior aspects of the trunk, neck and head traversed by this main channel become ninety degrees to the mid-line plane of the lower limb. However the pose chosen for the Ming dynasty drawing of the Bladder main channel was presented in a strikingly different manner - see Figure 10 example 4 and Figure 11. Here the trunk is turned to the right with the legs placed very far apart; while the head was rotated firmly to the left. This stance required considerable suppleness but does succeed in bringing all surfaces of the Bladder main channel to forty-five degrees to the mid-line plane of the leg.
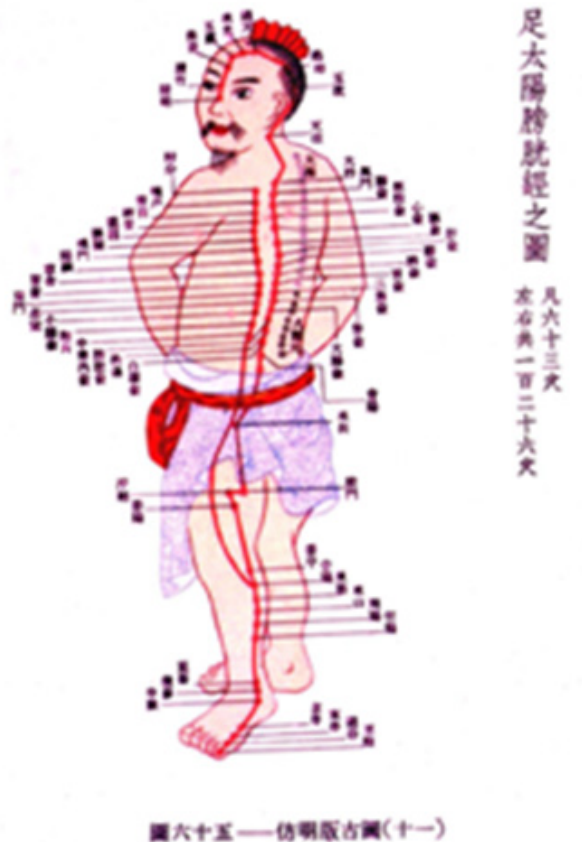

Figure I I Ming dynasty painting of a pose designed to reveal the foot, taiyang (great yang), posterolateral Bladder (BL) 'main channel' or 'meridian'. In this individual it appears to be arising from not from the upper margin of the orbit as shown in later drawings but the surface of his nose.

\section{Could the paths of main channels have been made visible?}

When the human body is entirely covered in a reflective substance such as oil and posed in the same way as the Ming Dynasty drawings see Figures $9 \& 10$ a 'line' of brightly reflected light can be observed along many of parts of the body. This is perceived as a 'line' travelling along regions of the body that are highly curved in cross-section such as the forearm; but where a relatively flat region occurs such as the side of the hand, the highlight becomes a good deal more spread out.

The number of lines seen on the body in this way at any one time depends on the number of light sources. To make these observations the subject covered in oil has to be presented in an otherwise completely dark room lit by only one lamp.

To find a pose that revealed a particular main channel, the light bulb was placed at the eye level of the observer in the immediate vicinity of the camera and its flash gun. Once a particular pose begins to show a 'line' of brightly reflected light following as much of the path of a main channel as possible, a photograph is taken (Figure 13).

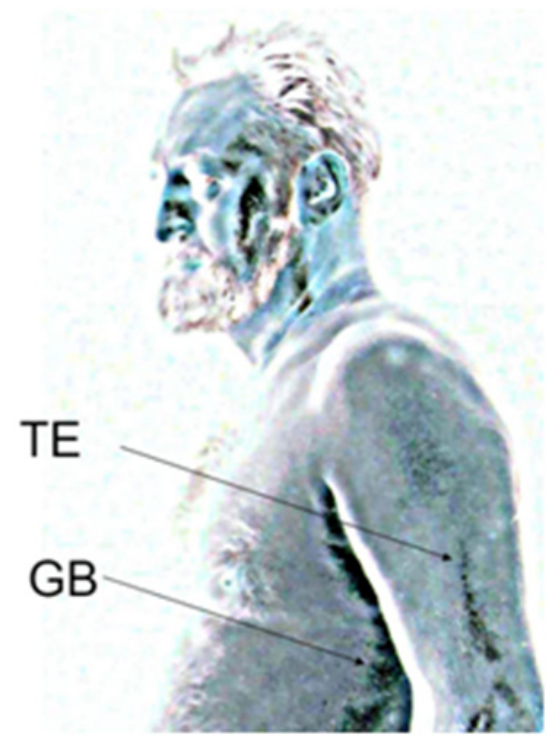

Figure 12 A photograph (in negative form taken by the author) of a pose where the lateral, shaoyang (lesser yang) 'main channels' or 'meridians' of the hand, triple energizer (TE) and the foot, gallbladder (GB) can be seen as linear highlights that pass upwards from the trunk and arm to intermingle in regions of the side of the head and ear.
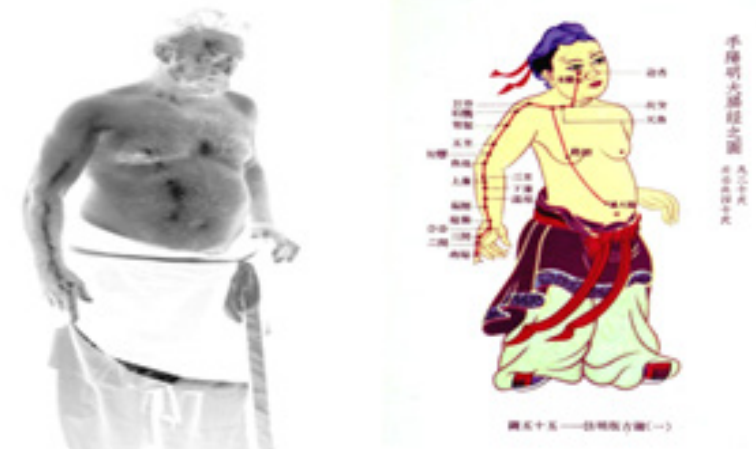

Figure I3 On the left a Ming Dynasty pose of the hand, anterolateral, yangming (sunlight yang) surface called the Large Intestine (LI) 'main channel'. On the right, a photograph (taken by the author, Macdonald 1982) in negative form of a figure entirely covered in oil posed in a similar manner in front of a single light source: here a dark line appears on the hand, forearm, neck, abdomen and head as shown in the Ming Dynasty drawing.

Many parts of main channels such as hand, lateral shaoyang (lesser yang) could be seen in several different poses of the upper limb, head and neck. In almost all cases, however, to render as much of the surface of any main channel visible at a time, I found the most convenient pose was suggested in the Ming Dynasty paintings, see Figures $9 \& 10$. Some of these poses, for example the Bladder and Liver, were not easy to imitate as they required the contortions of an acrobat. I also noted paths of the foot yin surfaces could be exhibited by the simple device of externally rotating the hip joint - see poses for the foot yin channels, Spleen, Liver and Kidney (Figure 10, examples 1,2 and 3 ).

Meanwhile various surfaces of the head itself are shared by yang main channels of foot and hand - see Figure 9examples 4, 5 and 6 and Figure 10 examples 4,5 and 6 . The anterior aspects of the head display surfaces of two foot yang main channels - Stomach and Bladder that appear to overlap, despite one being derived from the antero- while the other from the posterolateral foot yang surfaces; however the orbit 
provided a convenient site to separate them - one from its lower and the other from its upper margin.

Figure 12 is a photograph in negative form of a subject entirely covered in oil and lit from only one light source. Here we may see intermingling paths taken by the Gallbladder (GB) and Triple energizer (TE) that form the lateral shaoyang (lesser yang) surfaces of hand and foot in their progress over the surface of the head and pinna. To reveal as many details as possible and retain the anonymity of the subject, digital processing by Adobe Elements was employed to produce a negative. In this way, the highlights appear as dark lines as compared with surrounding regions. This technique has been employed here to indicate the highlights that could be perceived along surfaces of the regions of the oiled body placed in a similar pose to the Ming Dynasty painting of the hand, anterolateral, yangming (sunlight yang) surface of Large Intestine (LI) channel - see Figure 13: here more attention might have been paid to the position of the head, as this was not rotated enough to match the position recorded in the Ming Dynasty pose. Indeed while taking these photographs, it took an hour or so to find the correct pose to display as much of the course of a 'main channel' or 'meridian' as possible.

This leads one to suggest there is a possibility that these Ming dynasty Figure of main channels (Figures $9 \& 10$ ) were drawn from life, with subjects soaked in a light-reflective substance such as water, oil or sweat and posed in front of a single light source.

\section{Conclusion}

Why it was so important to study the paths of these particular surfaces of the body in such a manner is worthy of ongoing speculation. The main channel concepts may have been a scholarly way of explaining to patients why practitioners were looking for tender regions placed at some distance from where the patient was complaining of a referred pain, and the fact that a main channel was considered to travel from say the foot to the head may have recommended practising the non-segmental effects of noxious stimulation such as inserting an acupuncture needle a long distance away from the complaint.

Presenting the body's surfaces in this manner was not intended to indicate the anatomical paths of deeply lying structures but rather to present the observer with an understanding of something more functional in terms of the relationship of the body to the environment. For example, our understanding of the nervous system and withdrawal reflexes today suggests the effects of an acupuncture needle applied anywhere along at least some of the length of a surface of the body that presents in the same direction might well inhibit underlying agonist muscles and other neural inputs underlying that surface and facilitate the coordinated actions of antagonist muscles to withdraw the body from the noxious stimulus.

This hypothesis is in line with a suggestion by Zhao 2008: "Given that the meridian theory has been effectively used for treatment in traditional Chinese medicine, it is conceivable that the meridians might be a functional, but not an anatomical, concept that includes a summation of multiple physiological functions, including the nervous, circulatory, endocrine and immune systems. It is well known that the concept of the constellation has played an important role in astronomy and navigation for a long time. The meridian system might resemble the concept of the constellation in which fictive lines (channels) link various stars (acupoints)".

\section{Acknowledgments}

None.

\section{Conflicts of interest}

Author declares there are no conflicts of interest.

\section{Funding}

None.

\section{References}

1. Soulié de Morant G. Précis de la Vraie Acuponcture Chinoise. Paris; Mercure de France; 1934.

2. Research Group of Acupuncture Anesthesia, Institute of Medicine and Pharmacology of Fujian Province. Studies of phenomenon of blocking activities of channels and collaterals. In: Zhang X, Editor. Research on Acupuncture Moxibustion and Acupuncture Anesthesia. Beijing, Science Press; Springer Verlag, Berlin, Germany. 1986, p. 653-666.

3. Travell JG, Simons DG. Myofascial Pain and Dysfunction: the Trigger Point Manual - the Lower Extremities. Williams and Wilkins, USA. 1992.

4. Mann F. Scientific Aspects of Acupuncture. London: William Heinemann; 1997. p. 63

5. Melzack R, Stillwell DM, Fox EJ. Trigger points and acupuncture points for pain: correlations and implications. Pain. 1977;3(1):3-23.

6. Macdonald AJR. Acupuncture's non-segmental and segmental analgesic effects: the point of meridians. In: Filshie J, White A (Eds.), Medical Acupuncture: a Western Scientific Approach. UK: Churchill Livingstone; 1998. p. 83-104.

7. Dorsher PT. Myofascial referred-pain data provide physiologic evidence of acupuncture meridians. J Pain. 2009;10(7):723-731.

8. Langevin HM, Yandow JA. Relationship of acupuncture points and meridians to connective tissue planes. Anat Rec. 2002;269(6):252-265.

9. Bai Y, Wang J, Wu JP, et al. Review of evidence suggesting that the fascia network could be the anatomical basis for acupoints and meridians in the human body. Evidence-Based Complementary and Alternative Medicine. 2011;2011:260510.

10. Tao H, Yu MC, Yang HY, et al. Correlations between fasciology and Yin Yang doctrine. J Acupunct. Meridian Stud. 2011;4(2):141-146.

11. Schorrenberger CC. Anatomical roots of Chinese medicine and acupuncture. J Chin Med. 2008;19(1,2):35-63.

12. Wong KC, Wu L-T. History of Chinese Medicine. Tiensin, China: The Tientsin Press; 1932.

13. Eycleshymer AC, Schoemaker DM. A Cross-section Anatomy. USA: Meredith Corporation; 1970.

14. Mann F. Textbook of Acupuncture. USA: Heinemann' 1987.

15. Macdonald A. Acupuncture from Ancient Art to Modern Medicine. Australia: George Allen and Unwin; 1982.

16. WHO. Standard Acupuncture Nomenclature. Geneva: World Health Organization technical report series; 1984.

17. Zhao ZQ. Neural mechanism underlying acupuncture analgesia. Prog Neurobiol. 2008;85(4):355-375.

18. The Academy of Traditional Chinese Medicine. An Outline of Chinese Medicine. China: Foreign Languages Press; 1975. 